\title{
How to Leverage Collaborations Between the BME Community and Local Hospitals to Address Critical Personal Protective Equipment Shortages During the COVID-19 Pandemic
}

\author{
M. Patricia George, ${ }^{1}$ Lisa A. Maier, ${ }^{1}$ Shannon Kasperbauer, ${ }^{1}$ \\ JARED EdDy, ${ }^{1}$ AnNyCE S. Mayer, ${ }^{1}$ \\ and Chelsea M. Magin $\mathbb{1}^{2,3,4}$ \\ ${ }^{1}$ Department of Medicine, National Jewish Health, Denver, CO, USA; ${ }^{2}$ Department of Bioengineering, University of Colorado, \\ Denver $\mid$ Anschutz, Aurora, CO, USA; ${ }^{3}$ Department of Pediatrics, University of Colorado, Anschutz, Aurora, CO, USA; and \\ ${ }^{4}$ Division of Pulmonary Sciences and Critical Care Medicine, Department of Medicine, University of Colorado, Anschutz, \\ Aurora, CO, USA
}

(Received 2 July 2020; accepted 17 July 2020; published online 24 July 2020)

Associate Editor Joel Stitzel oversaw the review of this article.

\begin{abstract}
The global COVID-19 pandemic disrupted supply chains across the world, resulting in a critical shortage of personal protective equipment (PPE) for frontline healthcare workers. To preserve PPE for healthcare providers treating COVID-19 positive patients and to reduce asymptomatic transmission, the Department of Bioengineering at the University of Colorado, Denver | Anschutz Medical Campus collaborated with National Jewish Health to design and test patterns for cloth face coverings. A public campaign to sew and donate the final pattern was launched and over 2500 face coverings have been donated as a result. Now that nearly three million cases of COVID-19 have been confirmed in the United States, many state and local governments are requiring cloth face coverings be worn in public. Here, we present the collaborative design and testing process, as well as the final pattern for non-patient facing hospital workers and community members alike.
\end{abstract}

Keywords-COVID-19, Cloth face covering, Personal protective equipment.

\section{INTRODUCTION}

The World Health Organization (WHO) designated the COVID-19 outbreak as a global pandemic on March 11, 2020. By March 19, frontline healthcare workers already reported critical shortages of personal

Address correspondence to Chelsea M. Magin, Division of Pulmonary Sciences and Critical Care Medicine, Department of Medicine, University of Colorado, Anschutz, Aurora, CO, USA. Electronic mail: chelsea.magin@cuanschutz.edu protective equipment (PPE) including respiratory protection, gloves, face shields, gowns, and surgical masks. ${ }^{4}$ In the United States, the Food and Drug Administration (FDA) regulates medical respirators and surgical masks. These products must pass National Institute for Occupational Safety and Health (NIOSH) performance testing and be cleared for sale by submission of a premarket notification under section $510(\mathrm{k})$ of the Food, Drug and Cosmetic Act. Breakdown in the global supply chain of PPE and the resulting critical shortages led the FDA to release guidances allowing "use of improvised PPE when no alternatives, such as FDA-cleared masks or respirators, are available". 2 Simultaneously, the Centers for Disease Control and Prevention (CDC) recommended the use of face coverings by all people when out in public. $^{3}$ The official policy at National Jewish Health in Denver, Colorado focused on ensuring that certified PPE was preserved for frontline healthcare providers treating patients, including suspected or confirmed COVID-19 patients and other patients at high risk for contracting COVID-19. As a result, a significant need was identified to provide face coverings for a diverse group of non-patient-facing staff members, including non-clinical faculty, administrators, cafeteria workers, and valets. Local sewing groups began quickly ramping up efforts to produce and donate cloth face coverings in response. Unfortunately, these local volunteers did not realize that there are nuances that 
influence the efficacy of cloth face coverings that must be considered when selecting a pattern and materials.

To best address the need for an optimal cloth face covering design, a multidisciplinary collaboration among pulmonary medicine, occupational medicine, and bioengineering was formed to rapidly evaluate cloth face covering designs. A public campaign to produce cloth face coverings for staff members at the hospital using the approved design was launched within 10 days. In this letter, we describe the framework for introducing improvised PPE into a clinical setting during a crisis. We include previous studies that supported decisions for fabric type, the prototyping and fit-testing process, the feedback from occupational health experts and other physicians, and the final approved design. After sharing this pattern with our community, National Jewish Health received donations of over 2500 cloth face coverings for staff members in non-high-risk settings in an effort to reduce the risk of asymptomatic transmission.

\section{FRAMEWORK FOR COLLABORATIONS BETWEEN THE BME COMMUNITY AND LOCAL HOSPITALS}

Even though the design and production of improvised PPE does not require an engineering degree, it is critical that this product, like any other medical device, be constructed through close collaboration between the designers and the clinicians who will eventually use the product. Led by a faculty member in the University of Colorado, Denver I Anschutz Medical Campus Department of Bioengineering with experience in the medical device industry, a pulmonary and critical care physician working on the frontlines to treat COVID19 + patients, and an occupational medicine physician with expertise in respirator use and conservation both at National Jewish Health in Denver, our team produced prototypes of four publicly available cloth face covering patterns (Table 1).

All prototypes were constructed using two layers of tightly woven cotton cloth. The inner and outer layers were two visually different materials to ensure that wearers could easily tell the difference between the inside and outside of the mask. A study by Davies et al. evaluated the efficacy of common household materials in blocking transmission of bacterial and viral aerosols. ${ }^{1}$ Results showed that surgical masks filtered Bacteriophage MS2, the viral surrogate, with approximately $89 \%$ efficiency, while two layers of $100 \%$ cotton had a mean filtration efficiency of about $51 \%$. ${ }^{1}$ Based on these results and the availability of cotton fabric, we concluded that face coverings must be made from two layers of tightly woven, $100 \%$ cotton fabric, similar to what quilters use regularly.

The four prototypes were selected so that the occupational medicine physicians and respirator fit specialists at National Jewish Health could compare and evaluate three main design features: overall mask shape, conformation to the nasal bridge and jawline, and the method for securing the mask. The Deaconess (Fig. 1a) and We Can Sew It! (Fig. 1b) represent a pleated mask shape while the Florence Face Mask (Fig. 1c) and DIY Cloth Mask (Fig. 1d) designs are rounded. The We Can Sew It! and DIY Cloth Mask patterns featured the inclusion of a small wire along the nose bridge. The Deaconess and DIY Cloth Mask were both secured with elastic loops while the We Can Sew It! and Florence Face Mask designs were secured with fabric ties.

Prototypes were evaluated for seal, fit, and breathability. Feedback on the various design features is summarized in Table 2.

In response to this feedback, a designer and sewer selected the DIY Cloth Mask pattern, which featured a rounded mask shape and nasal bridge wire, and altered the original pattern to address concerns from the occupational medicine physicians and respirator fit specialists at National Jewish Health. The nose-to-chin length was increased, a short piece of elastic was added to the bottom for improved conformation to the jawline, and a novel system for securing the face covering using adjustable ties made from macramé cord or twill instead of static ties overcame the limitations of previous designs (Fig. 2). Ties that secured over the head allowed for a snugger fit than ear loop ties. This design was used to create a second round of prototypes

TABLE 1. Face covering designs selected for initial prototypes.

\begin{tabular}{lll}
\hline Design & \multicolumn{1}{c}{ Link to design tested } & Approver \\
\hline Deaconess & https://www.deaconess.com/How-to-make-a-Face-Mask & Deaconess Health System \\
We Can Sew It! & https://operationwecansewit.com/ & Saint Joseph Health \\
& SCL Health & N/A \\
Florence Face Mask & https://freesewing.org/blog/facemask-frenzy & N/A \\
DIY Cloth Face Mask & https://www.instructables.com/id/DIY-Cloth-Face-Mask/ & \\
\hline
\end{tabular}


(a)

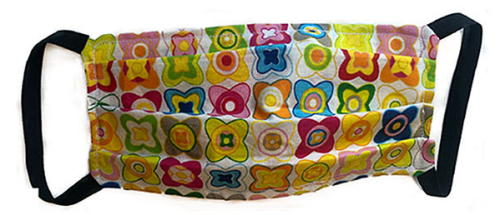

(c)

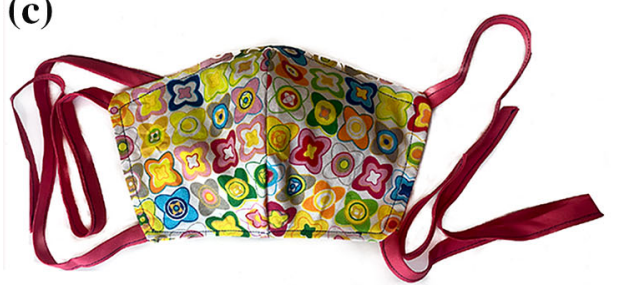

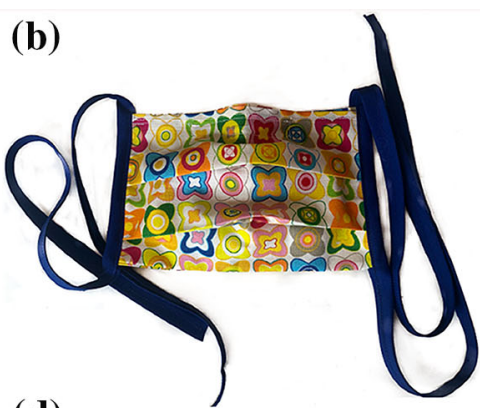

(d)

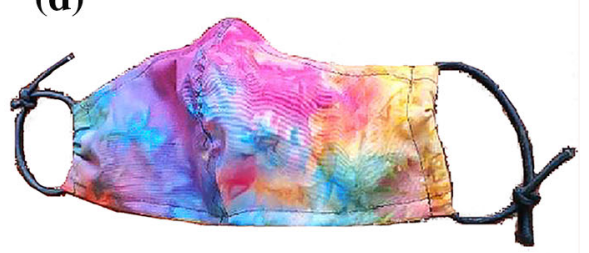

FIGURE 1. Representative photographs of prototype cloth face coverings that were sewn and delivered to National Jewish Health for testing including the (a) Deaconess, (b) We Can Sew It!, (c) Florence Face Mask, and (d) DIY Cloth Face Mask designs.

TABLE 2. Feedback on first round of prototypes.

Design feature

Pleated face covering shape

Rounded face covering shape

Nasal bridge wire

Elastic loops

Fabric ties

\section{Feedback}

Expansive, good coverage. Good breathability. Variable fit Good fit when nose-to-chin length was correct. Good breathability Improved fit for both prototypes

Loop size is critical and if it varies, the mask can be too tight or too loose Better for adjusting fit but loosened throughout the day. Over the head vs ear loop ties allowed for a snugger fit
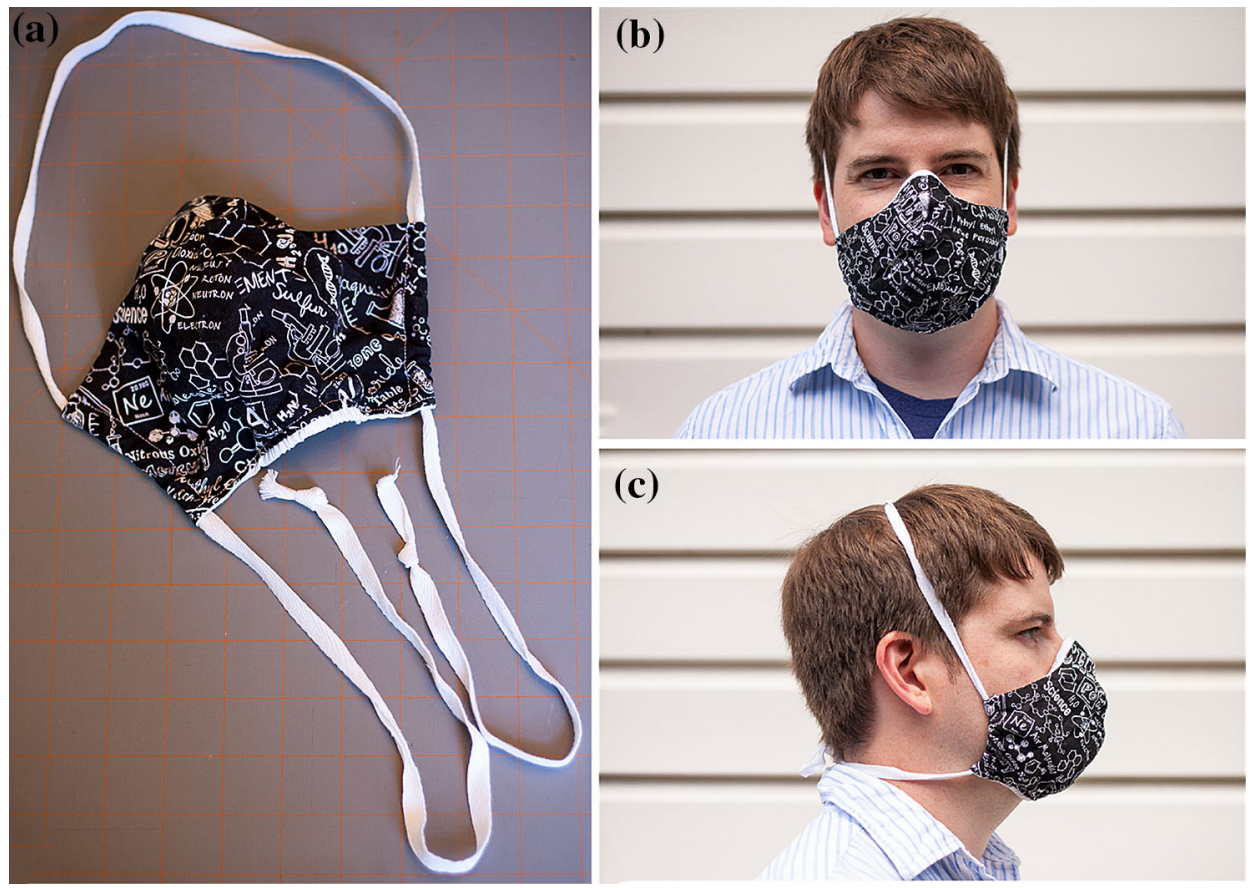

FIGURE 2. Photographs of (a) the final hospital-approved design, (b) the appropriate way to wear the face covering, and (c) secure the adjustable ties around the head. 
delivered to the hospital for evaluation. Ten volunteers, including doctors, nurses, and echocardiography technicians, wore these masks for at least $4 \mathrm{~h}$ before providing feedback. Overall comments were positive. Mask wearers remarked, "The mask fits great," and "I could wear it all day long and breathe".

\section{FINAL DESIGN AND PUBLIC CAMPAIGN}

We set out to design improvised PPE that could be fabricated locally by volunteers and would meet the requirements of a local hospital when supply chains failed to deliver certified face masks. The final design, evaluated and approved by the Occupational Medicine and Infectious Disease Groups at National Jewish Health, is available online (link: https://www.nationa ljewish.org/patients-visitors/patient-info/important-up dates/coronavirus-information-and-resources/additio nal-resources/making-masks-for-health-care-workers). This website includes a pattern and tutorial for sewing the approved design, best practices for wearing and maintaining cloth face coverings, answers to frequently asked questions, and instructions for making donations. The collaborative effort between the BME community and our local hospital described here resulted in the launch of the website and public campaign to sew and donate approved masks in fewer than 10 days. As of today, over 2500 cloth masks have been donated to National Jewish Health and provided to staff members in non-high-risk settings with the goal of reducing the risk of asymptomatic transmission of COVID-19. National Jewish Health has achieved and maintained face coverage for $100 \%$ of employees. Currently 46 states have some form of mandate requiring cloth face coverings be worn in public spaces. The design and materials outlined here are also ideal for community members to use in compliance with these requirements.

\section{ACKNOWLEDGMENTS}

The authors would like to thank Marilou Goodwin, Karen Gilbert, Seth Drake, Amber Platt, Jonathan Platt, Marti Freeman, Stephanie Alarcon, Anna Elmore, Lana Durkee, and Jason Vernon for their contributions to face covering design, sewing, and tutorial preparation. Thank you to David Wilson for demonstrating the proper way to wear cloth face coverings in Fig. 2.

\section{REFERENCES}

${ }^{1}$ Davies, A., K.-A. Thompson, K. Giri, G. Kafatos, J. Walker, and A. Bennett. Testing the efficacy of homemade masks: would they protect in an influenza pandemic? Disaster Med. Public Health Prepared. 7:413-418, 2013.

${ }^{2}$ Enforcement Policy for Face Masks and Respirators During the Coronavirus Disease (COVID-19) Public Health Emergency (Revised). In: Guidance for Industry and Food and Drug Administration Staff, edited by The U.S. Department of Health and Human Services Food and Drug Administration Center for Devices and Radiological Health.

${ }^{3}$ Centers for Disease Control and Prevention. Strategies for Optimizing the Supply of Facemasks. In: Coronavirus Disease 2019 (COVID-19), 2020.

${ }^{4}$ Ranney, M. L., V. Griffeth, and A. K. Jha. Critical supply shortages - the need for ventilators and personal protective equipment during the Covid-19 pandemic. New Engl. J. Med. 382:e41, 2020.

Publisher's Note Springer Nature remains neutral with regard to jurisdictional claims in published maps and institutional affiliations. 